\title{
Colletotrichum aracearum and C. camelliae-japonicae, two holomorphic new species from China and Japan
}

\author{
Hou LW ${ }^{1,2}$, Liu $F^{1}$, Duan WJ ${ }^{3}$, Cai $L^{1 *}$ \\ ${ }^{1}$ State Key Laboratory of Mycology, Institute of Microbiology, Chinese Academy of Sciences, Beijing 100101, China \\ ${ }^{2}$ University of Chinese Academy of Sciences, Beijing 100049, China \\ ${ }^{3}$ Ningbo Academy of Inspection and Quarantine, Ningbo 315012, China
}

Hou LW, Liu F, Duan WJ, Cai L 2016 - Colletotrichum aracearum and C. camelliae-japonicae, two holomorphic new species from China and Japan. Mycosphere 7(8) 1111-1123, Doi $10.5943 /$ mycosphere/si/2c/4

\begin{abstract}
Colletotrichum aracearum sp. nov. from Monstera delociosa and Philodenron selloum, and C. camelliae-japonicae sp. nov. from Camellia japonica, are described in this paper. Strains were isolated using single spore isolation technique from the spore masses formed on leaf lesions, and successfully induced for sexual states in cultivation. Morphological comparisons were made with related species. Phylogenetic relationships were inferred based on ITS, ACT, TUB2, and GAPDH sequences. $C$. camelliae-japonicae belongs to the $C$. boninense species complex, while $C$. aracearum does not nest within any currently known species complex but forms a sister clade to $C$. cliviae. This is the first report of Colletotrichum species from hosts Monstera delociosa and Philodenron selloum.
\end{abstract}

Key words - Anthracnose - Camellia - Monstera - Morphology - Philodenron - Phylogeny Taxonomy

\section{Introduction}

Colletotrichum is one of the most important genera among plant pathogenic fungi, mainly causing anthracnose on a wide range of crops or ornamental plants (Sutton 1980, Bailey et al. 1992, Freeman et al. 1998, Ureña-Padilla et al. 2002, Farr et al. 2006, Than et al. 2008a, Yang et al. 2009, Wikee et al. 2011, Noireung et al. 2012). Plant diseases associated with Colletotrichum species often caused significant economic losses (Sutton et al. 1992, Farr et al. 2006, Prihastuti et al. 2009). For example, Colletotrichum spp. cause extensive pre- and postharvest damage to chilli fruits, with yield losses up to 50\% (Manandhar et al. 1995, Pakdeevaraporn et al. 2005, Than et al. 2008b). In addition, many Colletotrichum species have also been recorded as endophytes, epiphytes, or saprobes (Photita et al. 2001, Kumar \& Hyde 2004, Liu et al. 2007, Prihastuti et al. 2009, Hyde et al. 2009, Rojas et al. 2010).

Studies of Colletotrichum could be traced back to Corda (1831), who first described Colletotrichum lineola. However, the taxonomy of Colletotrichum has been uncertain for centuries, until recently polyphasic characters, especially the molecular data were employed to delimitate species (Cannon et al. 2000, Hyde et al. 2009, Cai et al. 2009, Cannon et al. 2012, Damm et al. 2012b, Weir et al. 2012, Crouch 2014, Liu et al. 2015). Polyphasic characterization employing morphology and multi-locus phylogeny has contributed to the successful identification and epitypification of many Colletotrichum species which significantly stabilized the taxonomy (Damm 
et al. 2012a). Currently, Colletotrichum comprises of 11 major species complexes and several independent species. However, most species were only observed for their asexual morphs.

The objective of this study was to describe two novel Colletotrichum species from China and Japan for their holomorphic morphological characters. Phylogenetic relationships were inferred based on combined multi-locus sequence data.

\section{Materials \& Methods}

\section{Isolates and morphology}

Strains of Colletotrichum were isolated from the anthracnose lesions on Camellia japonica (camellia), Monstera delociosa (ceriman) and Philodendron selloum (lacy tree philodendron) from China and Japan. Single spore isolation technique was applied to plant tissue where spore masses were formed. Spore masses were picked off with a fine forceps and suspended in sterilized water. The spore suspension was diluted to a reasonable concentration and spread onto the surface potato dextrose agar (PDA), flowed by incubation overnight at room temperature $\left(25^{\circ} \mathrm{C}\right)$. Single germinating spores were picked up with a sterilized needle and transferred to new PDA plate for morphological and molecular study (Zhang et al. 2013).

Each isolate was plated onto PDA to prepare actively growing cultures. 4-mm-diam. plugs from the actively growing edge of a 5-day-old culture were transferred to the centre of fresh synthetic nutrient-poor agar medium (SNA) plates (Nirenberg 1976). The Anthriscus sylvestris stems were double-autoclaved and placed onto the surface of the SNA plate to promote sporulation (Damm et al. 2012a). Morphological descriptions were made after 7 days growth under alternating 12 hours near UV/12 hours dark at $25^{\circ} \mathrm{C}$ (Sutton 1980). Colony characters were observed and the diameter was measured at day seven. The growth rate was calculated as the 5-day average of mean daily growth (mm per day). Mycelial appressoria were produced using a slide culture technique (Sutton 1980). Microscopic preparations were made in clear lactic acid, with 30 measurements per structure as observed under a Nikon Eclipse 80i (Nikon Instech Company Limited, Kawasaki, Kanagawa, Japan) compound microscope using differential interference contrast (DIC) illumination. Taxonomic descriptions and nomenclature were deposited in Index Fungorum (Index Fungroum 2016).

\section{DNA extraction, PCR amplification and sequencing}

Genomic DNA of the isolates was extracted using the method of Guo et al. (2000). Four loci were amplified, including the internal transcribed spacer regions and intervening 5.8S rRNA gene (ITS), partial beta-tubulin (TUB2), actin (ACT), the glyceraldehyde-3-phosphate dehydrogenase (GAPDH), using primer pairs ITS1/ITS4, T1/Bt-2b, ACT-512F/ACT-783R, GDF1/GDR1, respectively. Amplification mixtures and conditions were followed as described by Liu et al. (2012). Purification and sequencing of PCR amplicons were carried out by the Biomed Sequencing Company, Beijing, China.

\section{Sequence alignment and molecular phylogenetic analysis}

Sequences of references and outgroup (Monilochaetes infuscans) were downloaded from GenBank and are listed in Table 1. Single gene and concatenated gene datasets were aligned with MAFFT v.7 (Katoh \& Frith 2012), and manually edited in MEGA v.6.0 when necessary (Tamura et al. 2013). Bayesian inference (BI) and Maximum Likelihood (ML) methods were implemented in this study. Bayesian analyses were performed using MrBayes v.3.2.2 (Ronquist et al. 2012) as described by Liu et al. (2014). Evolutionary models were selected by MrModeltest v.2.3 (Nylander 2004), with critical values for the topological convergence diagnostic set to 0.01. Posterior probabilities (PP) were determined by Markov Chain Monte Carlo sampling (BMCMC) in MrBayes v.3.2.2 (Zhaxybayeva \& Gogarten 2002, Ronquist et al. 2012). Four simultaneous Markov chains were run for 1000000 generations and trees were sampled every $100^{\text {th }}$ generation 
Table 1 Strains of Colletotrichum used in this study. Details are provided about hosts, location and GenBank accessions of the sequences generated

\begin{tabular}{|c|c|c|c|c|c|c|c|}
\hline \multirow[b]{2}{*}{ Species } & \multirow[b]{2}{*}{ Association number } & \multirow[b]{2}{*}{ Host } & \multirow[b]{2}{*}{ Locality } & \multicolumn{4}{|c|}{ GenBank accessions } \\
\hline & & & & ITS & GAPDH & ACT & TUB2 \\
\hline C. annellatum & CBS $129826^{*}$ & Hevea indica & Colombia & JQ005222 & JQ005309 & JQ005570 & JQ005656 \\
\hline C. araceaerum & CGMCC 3.14982, LC1033* & Monstera deliciosa & China & KX853166 & KX893585 & KX893577 & KX893581 \\
\hline C. araceaerum & CGMCC 3.14983, LC1041 & Philodendron selloum & China & KX853167 & KX893586 & KX893578 & KX893582 \\
\hline C. beeveri & CBS 128527, ICMP 18594* & Brachyglottis repanda & New Zealand & JQ005171 & JQ005258 & JQ005519 & JQ005605 \\
\hline C. boninense & CBS 123755, MAFF $305972 *$ & Crinum asiaticum & Japan & JQ005153 & JQ005240 & JQ005501 & JQ005588 \\
\hline C. boninense & CBS 128547, ICMP 10338 & Camellia sp. & New Zealand & JQ005159 & JQ005246 & JQ005507 & JQ005593 \\
\hline C. boninense & CBS 128526, ICMP 18591 & Dacrycarpus dacrydioides & New Zealand & JQ005162 & JQ005249 & JQ005510 & JQ005596 \\
\hline C. brasiliense & CBS 128501, ICMP 18607* & Passiflora edulis & Brazil & JQ005235 & JQ005322 & JQ005583 & JQ005669 \\
\hline C. brasiliense & CBS 128528, ICMP 18606 & Passiflora edulis & Brazil & JQ005234 & JQ005321 & JQ005582 & JQ005668 \\
\hline C. brassicicola & CBS 101059, LYN 16331* & Brassica oleracea & New Zealand & JQ005172 & JQ005259 & JQ005520 & JQ005606 \\
\hline C. brevisporum & BCC $38876^{*}$ & Neoregalia sp. & Thailand & JN050238 & JN050227 & JN050216 & JN050244 \\
\hline C. brevisporum & MFLUCC 100182 & Pandanus pygmaeus & Thailand & JN050239 & JN050228 & JN050217 & JN050245 \\
\hline C.camelliae-japonicae & CGMCC3.18117, LC6415 & Camellia japonica & Japan & KX853164 & KX893583 & KX893575 & KX893579 \\
\hline C.camelliae-japonicae & CGMCC3.18118, LC6416* & Camellia japonica & Japan & KX853165 & KX893584 & KX893576 & KX893580 \\
\hline C. citricola & CBS 134228 , CGMCC $3.15227^{*}$ & Citrus unshiu & China & KC293576 & KC293736 & KC293616 & KC293656 \\
\hline C. citricola & CBS 134229 & Citrus unshiu & China & KC293577 & KC293737 & KC293617 & KC293657 \\
\hline C. citricola & CBS 134230 & Citrus unshiu & China & KC293578 & KC293738 & KC293618 & KC293658 \\
\hline C. cliviae & CBS $125375^{*}$ & Clivia miniata & China & JX519223 & JX546611 & JX519240 & JX519249 \\
\hline C. colombiense & CBS 129818* & Passiflora edulis & Colombia & JQ005174 & JQ005261 & JQ005522 & JQ005608 \\
\hline C. colombiense & CBS 129817 & Passiflora edulis & Colombia & JQ005173 & JQ005260 & JQ005521 & JQ005607 \\
\hline C. constrictum & CBS 128504, ICMP 12941* & Citrus limon & New Zealand & JQ005238 & JQ005325 & JQ005586 & JQ005672 \\
\hline C. constrictum & CBS 128503, ICMP 12936 & Solanum betaceum & New Zealand & JQ005237 & JQ005324 & JQ005585 & JQ005671 \\
\hline C. curcumae & IMI 288937 & Curcuma longa & India & GU227893 & GU228285 & GU227991 & GU228187 \\
\hline C. cymbidiicola & IMI $347923 *$ & Cymbidium sp. & Australia & JQ005166 & JQ005253 & JQ005514 & JQ005600 \\
\hline C. cymbidiicola & CBS 128543, ICMP 18584 & Cymbidium sp. & New Zealand & JQ005167 & JQ005254 & JQ005515 & JQ005601 \\
\hline C. cymbidiicola & CBS 123757, MAFF 306100 & Cymbidium sp. & Japan & JQ005168 & JQ005255 & JQ005516 & JQ005602 \\
\hline C. dacrycarpi & CBS 130241, ICMP 19107* & Dacrycarpus dacrydioides & New Zealand & JQ005236 & JQ005323 & JQ005584 & JQ005670 \\
\hline C. dracaenophilum & CBS $118199 *$ & Buxus sp. & China & JX519222 & JX546707 & JX519238 & JX519247 \\
\hline
\end{tabular}


Table 1 (continued)

\begin{tabular}{|c|c|c|c|c|c|c|c|}
\hline C. excelsum-altitudum & CGMCC 3.15130, LC2344 & Bletilla ochracea & China & HM751815 & KC843502 & KC843548 & JX625211 \\
\hline C. excelsum-altitudum & CGMCC 3.15131, LC2345 & Bletilla ochracea & China & JX625182 & KC843503 & KC843549 & JX625212 \\
\hline C. gloeosporioides & IMI 356878*, CBS 112999 & Citrus sinensis & Italy & JX010152 & JX010056 & JX009531 & JX010445 \\
\hline C. karstii & CBS 129833 & Musa sp. & Mexico & JQ005175 & JQ005262 & JQ005523 & JQ005609 \\
\hline C. karstii & CBS 127591 & Sapium integerrimium & Australia & JQ005186 & JQ005273 & JQ005534 & JQ005620 \\
\hline C. karstii & CBS 132134, CGMCC 3.14194* & Vanda sp. & China & HM585409 & HM585391 & HM581995 & HM585428 \\
\hline C. novae-zelandiae & CBS 128505, ICMP 12944* & Capsicum аппиит & New Zealand & JQ005228 & JQ005315 & JQ005576 & JQ005662 \\
\hline C. novae-zelandiae & CBS 130240, ICMP 12064 & Citrus grapefruit & New Zealand & JQ005229 & JQ005316 & JQ005577 & JQ005663 \\
\hline C. oncidii & CBS $129828^{*}$ & Oncidium sp. & Germany & JQ005169 & JQ005256 & JQ005517 & JQ005603 \\
\hline C. oncidii & CBS 130242 & Oncidium sp. & Germany & JQ005170 & JQ005257 & JQ005518 & JQ005604 \\
\hline C. parsonsiae & CBS 128525, ICMP 18590* & Parsonsia capsularis & New Zealand & JQ005233 & JQ005320 & JQ005581 & JQ005667 \\
\hline C. petchii & CBS 118193, AR 3658 & Dracaena sanderana & China & JQ005227 & JQ005314 & JQ005575 & JQ005661 \\
\hline C. petchii & CBS 125957 & Dracaena sp. & Netherlands & JQ005226 & JQ005313 & JQ005574 & JQ005660 \\
\hline C. petchii & CBS 378.94* & Dracaena marginata & Italy & JQ005223 & JQ005310 & JQ005571 & JQ005657 \\
\hline C. phyllanthi & CBS 175.67, MACS $271 *$ & Phyllanthus acidus & India & JQ005221 & JQ005308 & JQ005569 & JQ005655 \\
\hline C. sp. & CBS 123921, MAFF 238642 & Dendrobium kingianum & Japan & JQ005163 & JQ005250 & JQ005511 & JQ005597 \\
\hline C. torulosum & CBS 128544, ICMP 18586* & Solanum melongena & New Zealand & JQ005164 & JQ005251 & JQ005512 & JQ005598 \\
\hline C. torulosum & CBS 102667 & Passiflora edulis & New Zealand & JQ005165 & JQ005252 & JQ005513 & JQ005599 \\
\hline C. tropicicola & BCC $38877 *$ & Citrus maxima & Thailand & JN050240 & JN050229 & JN050218 & JN050246 \\
\hline C. tropicicola & MFLUCC 100167 & Panphiopedilum bellatolum & Thailand & JN050241 & JN050230 & JN050219 & JN050247 \\
\hline C. truncatum & CBS $151.35^{*}$ & Phaseolus lunatus & USA & GU227862 & GU228254 & GU227960 & GU228156 \\
\hline C. truncatum & CBS 120709 & Capsicum frutescens & India & GU227877 & GU228269 & GU227975 & GU228171 \\
\hline C. yunnanense & AS 3.9167, CBS 132135* & Buxus sp. & China & JX546804 & JX546706 & JX519239 & JX519248 \\
\hline M. infuscans & CBS 869.96 & Ipomoea batatas & South Africa & JQ005780 & JX546612 & JQ005843 & JQ005864 \\
\hline
\end{tabular}

${ }^{\text {a }}$ AS, CGMCC: China General Microbiological Culture Collection; BCC: BIOTEC Culture Collection, Thailand; CBS: Culture collection of the Centraal bureau voor Schimmelcultures, Fungal Biodiversity Centre, Utrecht, The Netherlands; ICMP: International Collection of Microorganisms from Plants, Auckland, New Zealand; LC: Working collection of Lei Cai, housed at CAS, China; MACS: MACS Collection of Microorganisms, India; MAFF: Ministry of Agriculture, Forestry and Fisheries, Tsukuba, Japan; MFLUCC: Mae Fah Luang University Culture Collection, Chiang Rai, Thailand.

${ }^{*}=$ ex-type culture. Strains/sequences studied in this paper are in bold font. 
(resulting in 10000 total trees). The first $25 \%$ of the trees were discarded as the burn-in phase of each analysis, and the remaining trees were used to calculate posterior probabilities (Cai et al. 2006, Liu et al. 2012). Maximum-likelihood analyses including 1000 bootstraps replicates were conducted using RAxML v.7.2.6 (Stamatakis et al. 2010). A general time reversible model (GTR) was applied with a gamma-distributed rate variation. Trees were visualized in FigTree 1.4.0 (Rambaut 2012).

\section{Genealogical concordance phylogenetic species recognition analysis}

Phylogenetically closely related species were analyzed using the Genealogical Concordance Phylogenetic Species Recognition (GCPSR) model by performing a pairwise homoplasy index (PHI) test as described by Quaedvlieg et al. (2014). The PHI test was performed in SplitsTree v.4 (Huson 1998, Huson \& Bryant 2006) in order to determine the recombination level within phylogenetically closely related species using a 4-locus concatenated dataset (ACT, GAPDH, ITS and TUB2). Pairwise homoplasy index below a 0.05 threshold $\left(\Phi_{\mathrm{w}}<0.05\right)$ indicates significant recombination present in the dataset. The relationship between closely related species was visualized by constructing a splits graph.

\section{Results}

\section{Phylogenetic analyses}

Fifty-two combined ACT, GAPDH, ITS and TUB2 sequences were aligned, comprising 1730 characters including gaps after alignment (295 for ACT; 350 for GAPDH; 577 for ITS; 508 for TUB2). The concatenated alignment was deposited in TreeBASE (Study Accession URL: http://purl.org/phylo/treebase/phylows/study/TB2:S19933). For the Bayesian inference, a HKY+G model with inverse gamma distributed rate was selected for ACT; a HKY $+\mathrm{I}+\mathrm{G}$ model with inverse gamma distributed rate was selected for TUB2 and GAPDH; a GTR+I+G model with inverse gamma distributed rate was selected for ITS.

Two strains isolated from Camellia japonica clustered within the $C$. boninense complex, and the other two strains isolated from Philodenron selloum and Monstera delociosa formed a sister clade to $C$. cliviae, which do not belong to any previously known species complexes (Fig. 1).

Pairwise homoplasy index (PHI) test

A pairwise homoplasy index (PHI) test using a 4-gene dataset (ACT, GAPDH, ITS and TUB2) was performed to determine the recombination level between $C$. camelliae-japonicae and its phylogenetically closely related species, C. citricola (Fig. 2). No significant recombination events could be detected between $C$. camelliae-japonica and $C$. citricola $\left(\Phi_{\mathrm{W}=1}\right)$.

\section{Taxonomy}

Colletotrichum aracearum LW. Hou \& L. Cai, sp. nov.

Fig. 3

Index Fungorum number: IF552557

Etymology - named after the host plant family, Araceae.

Description - Asexual morph: Conidiomata acervular, pale yellow colored. Conidiophores and setae formed on a pale brown cushion. Setae 135-155 $\mu \mathrm{m}$ long, medium brown, smooth to verruculose, 3-4-septate, bacilliform, sometimes slightly inflated at base, 5-7 $\mu \mathrm{m}$ diam. at the widest part. Conidiophores hyaline or pale brown; mostly septate, branched or unbranched at the base, up to $52 \mu \mathrm{m}$ long. Conidiogenous cells hyaline or pale brown, cylindrical, ovoid, ampulliform or lageniform, 8.5-18 $\times$ 3-5 $\mu \mathrm{m}$. Conidia hyaline, oblong, apex and base rounded, often containing scattered small granular, wall smooth, aseptate, straight, 14-19 $\times 4.5-6 \mu \mathrm{m}$ (mean \pm $\mathrm{SD}=16.5 \pm 1.4 \times 5.7 \pm 0.3 \mu \mathrm{m}, \mathrm{n}=30), \mathrm{L} / \mathrm{W}$ ratio $=2.8$. Appressoria solitary, dark greenish, thick-walled, entire edge or crenate, rarely lobate, smooth-walled, ellipsoidal or irregular in shape, 7.5-12.5 $\times 5.5-9.5 \mu \mathrm{m}($ mean $\pm \mathrm{SD}=9.5 \pm 1.5 \times 7.4 \pm 1.2 \mu \mathrm{m}, \mathrm{n}=20), \mathrm{L} / \mathrm{W}$ ratio $=1.3$. 


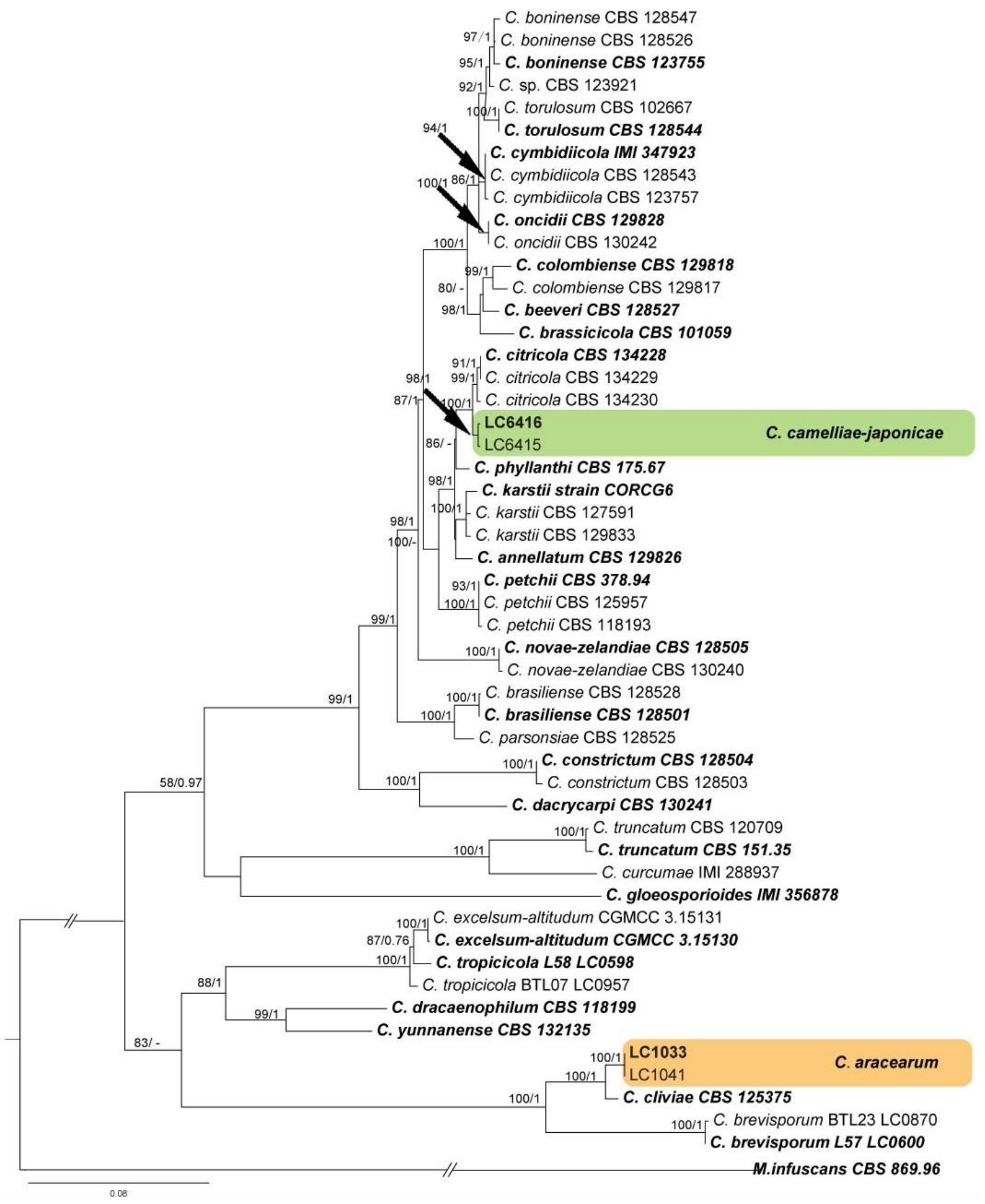

Fig. 1 - Phylogenetic tree from a Bayesian analysis based on combined gene sequences (ACT, GAPDH, ITS and TUB2) showing the phylogenetic relationships among the $C$. boninense complex, the $C$. truncatum complex, and independent units in Colletotrichum. The RAxML bootstrap support values (ML > 50) and Bayesian posterior probabilities ( $\mathrm{PP}>0.95)$ are displayed at the nodes (PP/ML). The tree is rooted with Monilochaetes infuscans (CBS 869.96). The scale bar indicates 0.08 expected changes per site. Ex-type cultures are emphasized in bold. 


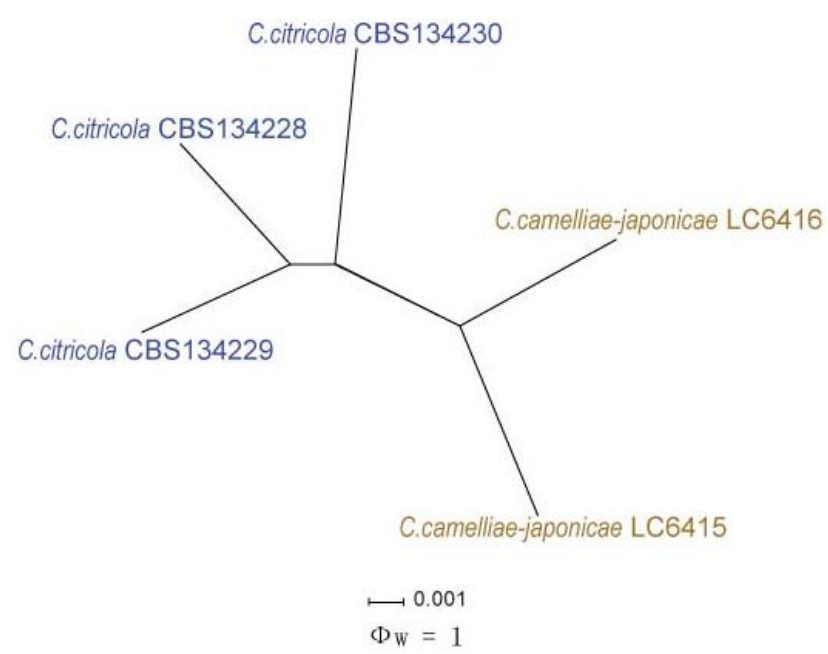

Fig. 2 - The result of the pairwise homoplasy index (PHI) test of closely related species using both LogDet transformation and splits decomposition. PHI test results $\left(\Phi_{\mathrm{w}}\right)<0.05$ indicate significant recombination within the dataset.

Sexual morph - Ascomata perithecia, oval, globose or obpyriform; medium brown, 175-260 $\times 125-165 \mu \mathrm{m}$; the outer wall composed of flattened angular cells $2.5-8.5 \mu \mathrm{m}$ diam. Interascal tissue composed of rather irregular thin-walled hyaline septate paraphyses. Asci in basal fascicle, clavate, with a truncated apex and a small refractive apical ring, 32.5-70 $\times 6$-14 $\mu \mathrm{m}, 8$-spored. Ascospores hyaline, aseptate, allantoid, 8.5-11.5 $\times 2-3 \mu \mathrm{m}$, mean $\pm \mathrm{SD}=10.2 \pm 0.9 \times 2.5 \pm 0.2$ $\mu \mathrm{m}, \mathrm{L} / \mathrm{W}$ ratio $=4.1$.

Culture characters - Colonies on SNA flat with entire margin, surface covered with dark brown or black ascomata and scattered pale yellow conidiomata. Surface of Anthriscus

covered with ascomata and partly with white mycelia. Reverse hyaline. Colonies on PDA attaining 6-6.2 cm diam. in 5 days at $25^{\circ} \mathrm{C}$, growth rate $12-13 \mathrm{~mm}$ per day; at first white, becoming pale grayish, finally become dark greenish with white edges; surface with dark ascomata. Reverse brown or greenish.

Materials examined - China, Guangdong Province, botanical garden, on Monstera deliciosa, 25 November 2010, Yuanying Su, holotype HMAS 243485, ex-holotype living culture CGMCC 3.14982 (= LC1033). China, Guangdong Province, botanical garden, on Philodenron selloum, Yuanying Su, 25 November 2010, living culture CGMCC 3.14983 (= LC1041).

Notes - Two strains of $C$. araceaerum were obtained from different hosts, i.e. Monstera delociosa and Philodenron selloum, both belonging to Araceae. Both strains presented similar growth rates and morphological characters. This is the first report of Colletotrichum from these two host plants (Farr \& Rossman 2016). The clade representing $C$. araceaerum is closely related to $C$. cliviae, a species that has not been observed for sexual morph (Yang et al. 2009). C. araceaerum differs from $C$. cliviae in producing shorter conidia $(14-19 \times 4.5-6 \mu \mathrm{m}$ vs. $19.5-24.5 \times 4.5-7$ $\mu \mathrm{m})$, smaller appresorria $(9.5 \pm 1.5 \times 7.4 \pm 1.2 \mu \mathrm{m}$ vs. $11.7 \pm 1.2 \times 8.6 \pm 1.2 \mu \mathrm{m})$, and slower growth rate $(12-13 \mathrm{~mm} /$ day vs. $15.2-16 \mathrm{~mm} /$ day $)$.

Colletotrichum camelliae-japonicae LW. Hou \& L. Cai, sp. nov. Index Fungorum Number: IF552558

Fig. 4

Etymology - named after the epithet of its host plants, Camellia japonica.

Description - Asexual morph: Vegetative hyphae 1.5-3 $\mu \mathrm{m}$ diam.; hyaline, smooth-walled, branched with septate. Conidiomata acervular, conidiophores formed on a pale brown cushion; conidiophores hyaline, septate, occasionally branched at base, 35.5-46 $\mu \mathrm{m}$ long. Setae unobserved. Conidiogenous cells hyaline, 7-21.5 $\times 3-4.5 \mu \mathrm{m}$, oblong to ampulliform, often extending to form new conidiogenous loci. Conidia hyaline, oblong, single-celled, apex and base rounded, with a 


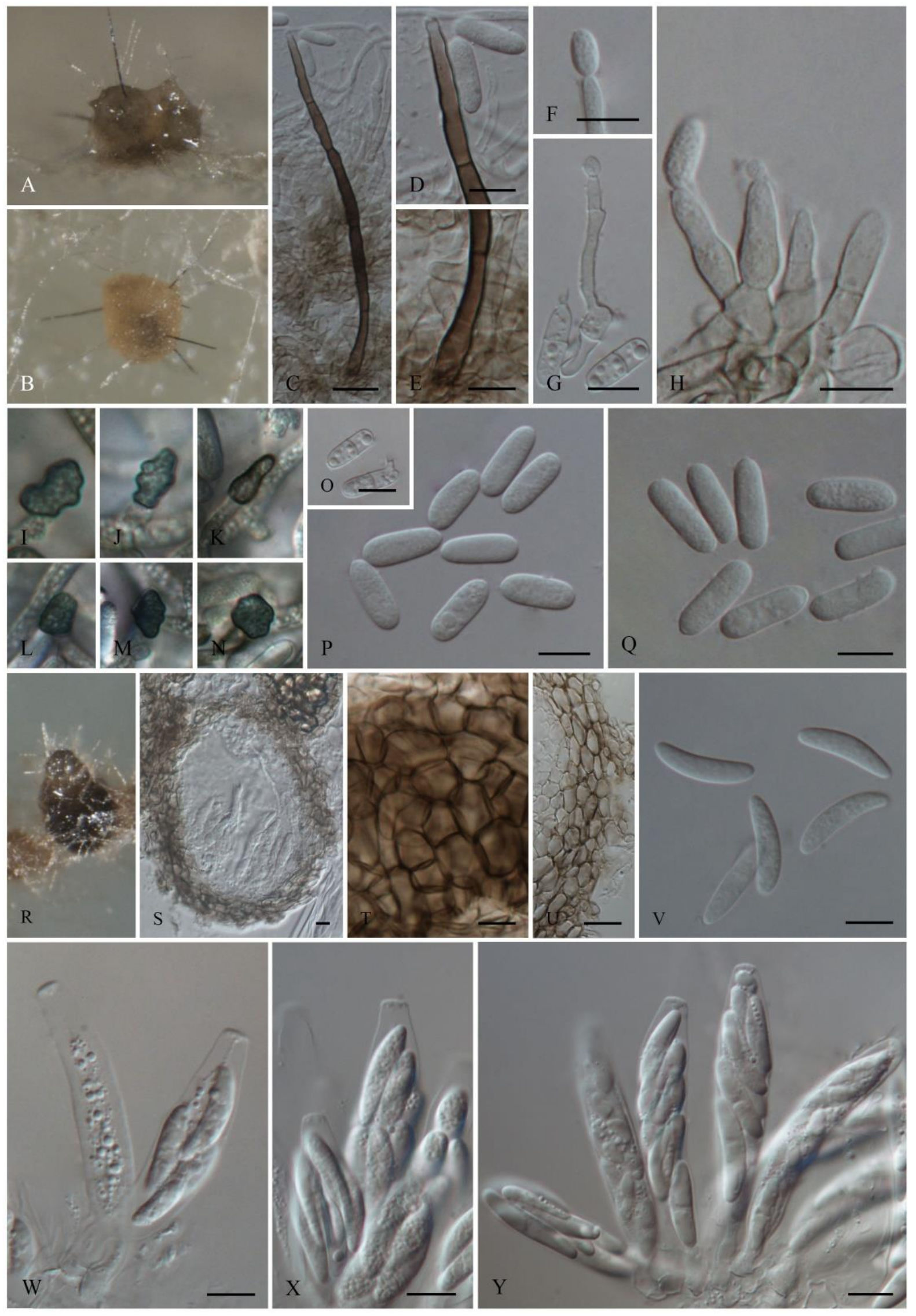

Fig. 3 - Colletotrichum araceaerum (from ex-holotype strain LC1033) on SNA. A, B Conidiomata. C, D, E Setae. F, G, H Conidiophores. I, J, K, L, M, N Appressoria. O, P Conidia from SNA. Q Conidia from the Anthriscus sylvestris stems. R, S Ascomata. T Outer surface of apical regions of asci. U Peridium in cross section. V Ascospores. W, X, Y Asci. - Scale bars $=10 \mu \mathrm{m}$. 

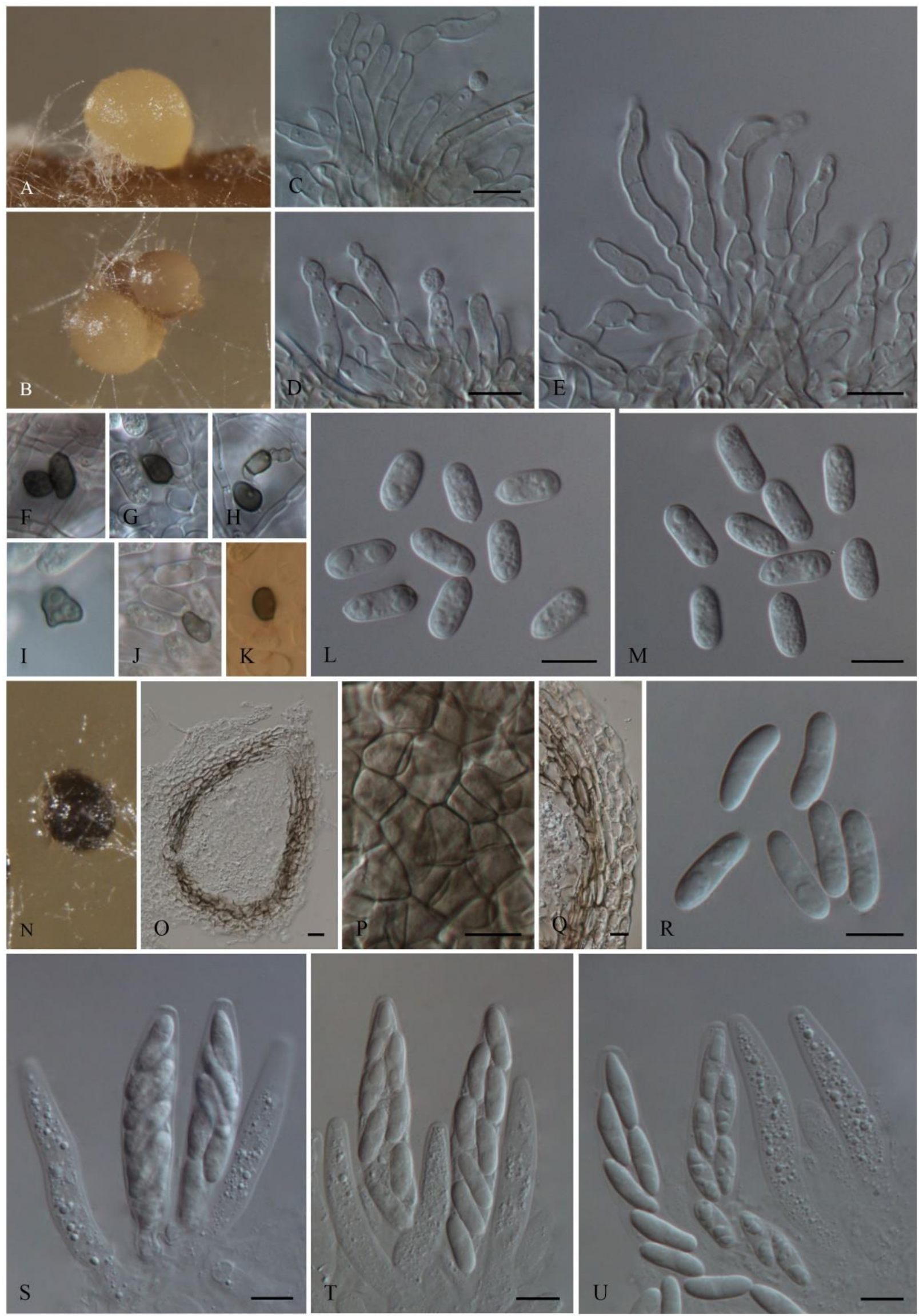

Fig. 4 - Colletotrichum camelliae-japonicae (from holotype strain LC6416) on SNA. A, B Conidiomata. C, D, E Conidiophores. F, G, H, I, K Appressoria. L Conidia from SNA. M Conidia from the Anthriscus sylvestris stems. N, O Ascomata. P Outer surface of apical regions of asci. Q Peridium in cross section. R Ascospores. S, T, U Asci. - Scale bars $=10 \mu \mathrm{m}$. 
prominent scar; smooth-walled, aseptate, most contents granular or guttulate; $11-14.5 \times 5-6.5 \mu \mathrm{m}$, mean $\pm \mathrm{SD}=12.5 \pm 0.8 \times 5.5 \pm 0.3 \mu \mathrm{m}, \mathrm{L} / \mathrm{W}$ ratio $=2.3$. Appressoria solitary, dark greenish, thick-walled, entire edge, subglobose, ellipsoidal or obpyriform, sometimes irregular in shape, smooth-walled, 5-11 $\times 4-7 \mu \mathrm{m}$ (mean $\pm \mathrm{SD}=8.5 \pm 1.6 \times 5.5 \pm 0.9 \mu \mathrm{m}, \mathrm{n}=20), \mathrm{L} / \mathrm{W}$ ratio $=1.5$.

Sexual morph - Ascomata perithecia, ellipsoidal, globose, ovoid, obpyriform, aggregated or scattered, 190-250 × 155-250 $\mu \mathrm{m}$, glabrous, brown, abundant but mostly sterile. The outer wall of ascomata composed of flattened angular cells, 3-5.5 $\mu \mathrm{m}$ diam. Interascal tissue composed of rather irregular thin-walled hyaline septate paraphyses. Asci clavate, 58.5-79 $\times 11.5-14.5 \mu \mathrm{m}$ long, 8spored; apex with a truncated and a small apical ring. Ascospores hyaline, one-celled, allantoid or fusiform, slightly curved, $13.5-18.5 \times 4-5.5 \mu \mathrm{m}$, mean $\pm \mathrm{SD}=16.5 \pm 1.1 \times 5 \pm 0.4 \mu \mathrm{m}, \mathrm{L} / \mathrm{W}$ ratio $=3.3$.

Culture characters - Colonies on SNA flat, lacking aerial mycelium; surface covered with orange or pale yellow conidiomata; surface of Anthriscus covered with ascomata and white mycelium. Reverse hyaline. Colonies on PDA attaining 4-4.2 cm diam. in 7 days at $25{ }^{\circ} \mathrm{C}$, growth rate $8-9 \mathrm{~mm}$ per day; at first white, becoming grayish and finally covered with orange conidia mass. Reverse pale brown or grayish.

Material examined - Japan, intercepted by Ningbo Entry-Exit Inspection and Quarantine Bureau when exporting to China, on Camellia japonica, Weijun Duan, 25 November 2013, HMAS 247042 (Holotype designated here), ex-holotype living culture CGMCC 3.18118 (=LC6416); ibid. CGMCC 3.18117 (=LC6415).

Notes - Two strains of $C$. camelliae-japonicae were isolated from Camellia japonica imported from Japan and intercepted by Ningbo Entry-Exit Inspection and Quarantine Bureau. Camellia japonica is a commonly cultivated economic crop in China and other Asian countries. Several species of Colletotrichum have been reported as pathogens and endophytes from Camellia (Liu et al. 2015), but C. camelliae-japonicae is distinct from known species in morphological and phylogenetic characters. Based on multi-locus sequence data (ACT, GAPDH, ITS and TUB2), the clade representing $C$. camelliae-japonicae did not nest in any known species complexes but formed a sister clade to $C$. citricola with highly supported bootstrap value and posterior probability (Fig. 1). Colletotrichum camelliae-japonicae differs from $C$. citricola in producing narrower ascospores (4.0-5.5 $\mu \mathrm{m}$, mean $\pm \mathrm{SD}=5.0 \pm 0.4 \mu \mathrm{m}$ vs. 5.3-6.7 $\mu \mathrm{m}$, mean $=6.1 \mu \mathrm{m}, \mathrm{L} / \mathrm{W}$ ratio 2.6 vs. 3.3) and shorter conidia $(11-14.5 \mu \mathrm{m}$, mean $\pm \mathrm{SD}=12.5 \pm 0.8 \mu \mathrm{m}$ vs. $13.7-16.1 \mu \mathrm{m}$, mean $=15.1 \mu \mathrm{m})$.

\section{Discussion}

Although the ITS has been proposed as a universal barcode for fungi (Begerow et al. 2010, Druzhinina et al. 2005, Eberhardt et al. 2010, Schoch et al. 2011, Schoch et al. 2012), it is evolutionarily too conserved to distinguish taxa in Colletotrichum (Du et al. 2005, Crouch et al. 2009, Begerow et al. 2010). In this study, polyphasic approach was used to identify strains of Colletotrichum. It is very interesting that both new species described in this study have been successfully induced for the sexual stages and described.

Clades representing two new species were well separated from known species in the phylogenetic tree. Both species also present distinct morphological differences from their closely related species. Apart from 11 major lineages in this genus, previous studies also revealed several independently evolved small clusters, which have been named C. dracaenophilum, C. yunnanense and C. cliviae (Cannon et al. 2012). Colletotrichum araceaerum appeared to be an additional distinct species that do not belong to the 11 major clades.

Plants belongs to the Araceae are frequently infected by Colletotrichum species (Farr \& Rossman 2016). This is the first report of Colletotrichum species from hosts Monstera delociosa and Philodenron selloum in Araceae. As economically important crops in Asia (Wachira et al. 1995), Camellia spp. have been widely cultivated from the Himalayas to Japan and Indonesia. As a potential quarantine object, accurate identification of $C$. camelliae-japonicae contributes to the prevention of the outbreak of camellia anthracnose. 


\section{Acknowledgements}

Yuanying $\mathrm{Su}$ is thanked for providing strains. This study was financially supported by the National Natural Science Foundation of China (NSFC 31322001). L.W. Hou acknowledges CAS QYZDB-SSW-SMC044 for supporting her postgraduate studentship.

\section{References}

Bailey JA, O'connell RJ, Pring RJ, Nash C, Jeger MJ. 1992 - Infection strategies of Colletotrichum species. In: Colletotrichum: Biology, Pathology and Control (Bailey JA, Jeger MJ, eds), CAB International, Wallingford, The United Kingdom 88-120.

Begerow D, Nilsson H, Unterseher M, Maier W. 2010 - Current state and perspectives of fungal DNA barcoding and rapid identification procedures. Applied Microbiology and Biotechnology 87, 99-108.

Cai L, Hyde KD, Taylor PWJ, Weir B, Waller J, Abang MM, Zhang JZ, Yang YL, Phoulivong S, Liu ZY, Prihastuti H, Shivas RG, McKenzie EHC, Johnston PR. 2009 - A polyphasic approach for studying Colletotrichum. Fungal Diversity 39, 183-204.

Cai L, Jeewon R, Hyde KD. 2006 - Phylogenetic investigations of Sordariaceae based on multiple gene sequences and morphology. Mycological Research 110, 137-150.

Cannon PF, Bridge PD, Monte E. 2000 - Linking the past, present and future of Colletotrichum systematics. In: Colletotrichum : Host Specificity, Pathology, and Host Pathogen Interaction (eds. D.Prusky, S. Freeman and M.B. Dickman). APS Press, St. Paul, Minnesota, USA: 1-20.

Cannon PF, Damm U, Johnston PR, Weir BS. 2012 - Colletotrichum-current status and future directions. Studies in Mycology 73, 181-213.

Corda ACI. 1831 - Die Pilze Deutschlands. In: Deutschlands Flora in Abbildungennach der Natur mit Beschreibungen (Sturm, J). Sturm, Nürnberg vol. 3, Abt. 12: 33-64, tab. 21-32.

Crouch JA, Clarke BB, Hillman BI. 2009 - What is the value of ITS sequence data in Colletotrichum systematics and species diagnosis? A case study using the falcate-spored graminicolous Colletotrichum group. Mycologia 101, 648-656.

Crouch JA. 2014 - Colletotrichum caudatum s. $l$. is a species complex. IMA Fungus 5, 17-30.

Damm U, Cannon PF, Woudenberg JHC, Crous PW. 2012a - The Colletotrichum acutatum species complex. Studies in Mycology 73, 37-113.

Damm U, Cannon PF, Woudenberg JHC, Johnston PR, Weir BS, Tan YP, Shivas RG, Crous PW. 2012b - The Colletotrichum boninense species complex. Studies in Mycology 73, 1-36.

Druzhinina IS, Kopchinskiy AG, Komoń M, Bissett J, Szakacs G, Kubicek CP. 2005 - An oligonucleotide barcode for species identification in Trichoderma and Hypocrea. Fungal Genetics and Biology 42, 813-828.

Du M, Schardl CL, Nuckles EM, Vaillancourt LJ. 2005 - Using mating-type gene sequences for improved phylogenetic resolution of Collectotrichum species complexes. Mycologia 97, 641658.

Eberhardt U. 2010 - A constructive step towards selecting a DNA barcode for fungi. New Phytologist 187, 265-268.

Farr DF, Aime MC, Rossman AY, Palm ME. 2006 - Species of Colletotrichum on Agavaceae. Mycology Research 110, 1395-1408.

Farr DF, Rossman AY. 2016 - Fungal Databases, Systematic Mycology and Microbiology Laboratory, ARS, USDA. http://nt.ars-grin.gov/fungaldatabases/ (Retrieved August 7, 2016).

Freeman S, Katan T, Shabi E. 1998 - Characterization of Colletotrichum species responsible for anthracnose diseases of various fruits. Plant Disease, American Phytopathological Society, St. Paul, Minnesota, USA 82, 596-605.

Guo LD, Hyde KD, Liew ECY. 2000 - Identification of endophytic fungi from Livistona chinensis based on morphology and rDNA sequences. New Phytologist 147, 617-630.

Huson DH, Bryant D. 2006 - Application of phylogenetic networks in evolutionary studies. Molecular Biology and Evolution 23, 254-267. 
Huson DH. 1998 - SplitsTree: analyzing and visualizing evolutionary data. Bioinformatics 14, 6873.

Hyde KD, Cai L, McKenzie EHC, Yang YL, Zhang JZ, Prihastuti H. 2009 - Colletotrichum: a catalogue of confusion. Fungal Diversity 39, 1-17.

Index Fungorum. $2016 \quad-\quad$ Index Fungorum. Available from: http://www.indexfungorum.org/names/Names.asp (accessed 14 July 2016).

Katoh K, Frith MC. 2012 - Adding unaligned sequences into an existing alignment using MAFFT and LAST. Bioinformatics 28, 3144-3146.

Kumar DSS, Hyde KD. 2004 - Biodiversity and tissue-recurrence of endophytic fungi from Tripterygium wilfordii. Fungal Diversity 17, 69-90.

Liu F, Cai L, Crous PW, Damm U. 2014 - The Colletotrichum gigasporum species complex. Persoonia 33, 83-97.

Liu F, Hu DM, Cai L. 2012 - Conlarium duplumascospora gen. et. sp. nov. and Jobellisia guangdongensis sp. nov. from freshwater habitats in China. Mycologia 104, 1178-1186.

Liu F, Weir BS, Damm U, Crous PW, Wang Y, Liu B, Wang M, Zhang M, Cai L. 2015 Unravelling Colletotrichum species associated with Camellia: employing ApMat and GS loci to resolve species in the $C$. gloeosporioides complex. Persoonia 35, 63-86.

Liu XY, Xie XM, Duan JX. 2007 - Colletotrichum yunnanense sp. nov., a new endophytic species from Buxus sp. Mycotaxon 100, 137-144.

Manandhar JB, Hartman GL, Wang TC. 1995 - Anthracnose development on pepper fruits inoculated with Colletotrichum gloeoporioides. Plant Disease, American Phytopathological Society, St. Paul, Minnesota, USA 79, 380-383.

Nirenberg HI. 1976 - Untersuchungen uber die morphologische und biologische Differenzierung in der Fusarium-Sektion Liseola. Mitt. Biol. Bundesanst. Land-u. Forstwirtsch. Berlin-Dahlem, 169: 1-117.

Noireung P, Phoulivong S, Liu F, Cai L, Mckenzie EHC, Chukeatirote E, Jonese EBG, Ali H Bahkalif, Hyde KD. 2012 - Novel species of Colletotrichum revealed by morphology and molecular analysis. Cryptogamie, Mycologie 33, 347-362.

Nylander JAA. 2004 - MrModeltest v2.3 software. Evolutionary Biology Center, Uppsala University, Sweden.

Pakdeevaraporn P, Wasee S, Taylor PWJ, Mongkolporn O. 2005 - Inheritance of resistance to anthracnose caused by Colletotrichum capsici in Capsicum. Plant Breeding 124, 206-208.

Photita W, Lumyong S, Lumyong P, Ho WH, McKenzie EHC, Hyde KD. 2001 - Fungi on Musa acuminata in Hong Kong. Fungal Diversity 6, 99-106.

Prihastuti H, Cai L, Chen H, McKenzie EHC, Hyde KD. 2009 - Characterization of Colletotrichum species associated with coffee berries in northern Thailand Fungal Diversity 39, 89-109.

Quaedvlieg W, Binder M, Groenewald JZ, Summerell BA, Carnegie AJ, Burgess TI, Crous PW. 2014 - Introducing the Consolidated Species Concept to resolve species in the Teratosphaeriaceae. Persoonia-Molecular Phylogeny and Evolution of Fungi 33, 1-40.

Rambaut AA. 2012 - FigTree, version 1.4.0. http://tree.bio.ed.ac.uk/ software/ figtree/ (accessed 2012, December 5).

Rojas EI, Rehner SA, Samuels GJ, Van Bael SA, Herre EA, Cannon P, Chen R, Pang JF, Wang RW, Zhang YP, Peng YQ, Sha T. 2010 - Colletotrichum gloeosporioides sl. associated with Theobroma cacao and other plants in Panama: multilocus phylogenies distinguish hostassociated pathogens from asymptomatic endophytes. Mycologia 102, 1318-1338.

Ronquist F, Teslenko M, van der Mark P, Ayres DL, Darling A, Höhna S, Bret L, Liang L, Marc AS, Huelsenbeck JP. 2012 - MrBayes 3.2: efficient Bayesian phylogenetic inference and model choice across a large model space. Systematic Biology 61, 539-542.

Schoch C, Seifert K, Crous PW. 2011 - Progress on DNA barcoding of Fungi. IMA Fungus 2, 5-9.

Schoch C, Seifert K, Huhndorf S, Robert V, Spouge JL, Levesque CA, Chen W, Miller AN. 2012 Nuclear ribosomal internal transcribed spacer (ITS) region as a universal DNA barcode marker for Fungi. Proceedings of the National Academy of Sciences 109, 6241-6246. 
Stamatakis A, Alachiotis N. 2010 - Time and memory efficient likelihood-based tree searched on phylogenomic alignments with missing data. Bioinformatics 26, i132-i139.

Sutton BC, Bailey JA, Jeger MJ. 1992 - The genus Glomerella and its anamorph Colletotrichum. Colletotrichum: biology, pathology and control, CAB International, Wallingford, The United Kingdom 1-26.

Sutton BC. 1980 - The Coelomycetes: Fungi Imperfecti with Pycnidia Acervuli and Stomata. Commonwealth Mycological Institute, Kew, England.

Tamura K, Stecher G, Peterson D, Filipski A, Kumar S. 2013 - MEGA6: molecular evolutionary genetics analysis version 6.0. Molecular Biology and Evolution 30, 2725-2729.

Than PP, Jeewon R, Hyde KD, Pongsupasamit S, Mongkolporn O, Taylor PWJ. 2008a Characterization and pathogenicity of Colletotrichum species associated with anthracnose on chilli (Capsicum spp.) in Thailand. Plant Pathology 57, 562-572.

Than PP, Prihastuti H, Phoulivong S, Taylor PW, Hyde KD. 2008b - Chilli anthracnose disease caused by Colletotrichum species. Journal of Zhejiang University Science B 9, 764-778.

Ureña-Padilla AR, MacKenzie SJ, Bowen BW, Legard DE. 2002 - Etiology and population genetics of Colletotrichum spp. causing crown and fruit rot of strawberry. Phytopathology 92, $1245-1252$.

Wachira FN, Waugh R, Powell W, Hackett CA. 1995 - Detection of genetic diversity in tea (Camellia sinensis) using RAPD markers. Genome 38, 201-210.

Weir BS, Johnston PR, Damm U. 2012 - The Colletotrichum gloeosporioides species complex. Studies in Mycology 73, 115-180.

Wikee S, Cai L, Pairin N, McKenzie EH, Su YY, Chukeatirote E, Thi HN, Bahkali AH, Moslem MA, Abdelsalam K, Hyde KD. 2011 - Colletotrichum species from Jasmine (Jasminum sambac). Fungal Diversity 46, 171-182.

Yang YL, Liu ZY, Cai L, Hyde KD, Yu ZN, McKenzie EHC. 2009 - Colletotrichum anthracnose of Amaryllidaceae. Fungal Diversity 39, 123-146.

Zhang K, Su YY, Cai L. 2013 - An optimized protocol of single spore isolation for fungi. Cryptogamie, Mycologie 34, 349-356.

Zhaxybayeva O, Gogarten JP. 2002 - Bootstrap, Bayesian probability and maximum likelihood mapping: exploring new tools for comparative genome analyses. Genomics 3, 1-15. 